\begin{tabular}{|l|l|l|}
\hline \multicolumn{2}{|c|}{ PublisherInfo } \\
\hline \hline PublisherName & $:$ & BioMed Central \\
\hline \hline PublisherLocation & $:$ & London \\
\hline \hline PublisherImprintName & $:$ & BioMed Central \\
\hline \hline
\end{tabular}

\title{
Purification and cloning of aggrecanase-1
}

\begin{tabular}{||l|l|l||}
\hline \multicolumn{2}{|c||}{ ArticleInfo } \\
\hline \hline ArticleID & $:$ & 246 \\
\hline \hline ArticleDOI & $:$ & $10.1186 /$ ar-1999-66725 \\
\hline \hline ArticleCitationID & $:$ & 66725 \\
\hline \hline ArticleSequenceNumber & $:$ & 203 \\
\hline \hline ArticleCategory & $:$ & Paper Report \\
\hline \hline ArticleFirstPage & $:$ & 1 \\
\hline \hline ArticleLastPage & $:$ & 4 \\
\hline \hline & & RegistrationDate : 1999-9-2 \\
ArticleHistory & $:$ & OnlineDate \\
\hline \hline ArticleCopyright & $:$ & Current Science Ltd1999-9-2 \\
\hline \hline ArticleGrants & $:$ & \\
\hline \hline ArticleContext & $:$ & 130753311 \\
\hline \hline
\end{tabular}




\section{Keywords}

\section{Context}

Aggrecan is a major structural component of cartilage and forms large proteoglycan aggregates via interaction at its N-terminal G1 domain with hyaluronic acid and link protein. Proteolysis of the core protein of aggrecan can lead to loss of large glycosaminoglycan-containing aggrecan fragments from the tissue. There are two main sites of proteolytic cleavage in the interglobular domain (IGD) of the core protein, the matrix metalloproteinase (MMP) site between Asn341 and Phe342 and the "aggrecanase" site between Glu373 and Ala374. Fragments containing both N-terminal and C-terminal sequences that result from cleavage at these sites have been identified in conditioned medium from cartilage explants in which degradation was stimulated with interleukin (IL)-1 as well as in synovial fluids of patients with osteoarthritis, inflammatory joint disease and joint injury. Although a number of MMPs have been shown to cleave aggrecan at the MMP site, the identity of the "aggrecanase" has remained unknown for a number of years. The production of monoclonal antibodies that specifically detect the neoepitopes generated by proteolytic cleavage at each site has provided a useful assay system for monitoring the purification of "aggrecanase". This paper reports the identity and cloning of aggrecanase-1 from bovine nasal cartilage stimulated with IL-1. To purify and clone "aggrecanase", an enzyme which will cleave aggrecan between Glu373 and Ala374 in the interglobular domain.

\section{Significant findings}

SDS-PAGE analysis of the final fraction from the purification resulted in one predominant Coomassie-stained protein which migrated as a doublet at approximately $62 \mathrm{kDa}$. The protein doublet possessed aggrecanase activity comparable to that found in the initial conditioned medium. The Nterminal sequence and the sequence of two internal tryptic peptides revealed unique amino acid sequences with similarity to the murine protein ADAMTS-1 (a disintegrin and metalloproteinase with thrombospondin motifs). The internal amino acid sequences showed only one residue difference (out of 17 residues) compared to sequences encoded by a murine EST (expressed sequence tag). Using this EST as a starting point, a full length human cDNA encoding 837 amino acids was finally assembled. The enzyme contained a signal sequence, propeptide domain, potential furin cleavage site and cysteine 
switch, a catalytic domain, a disintegrin domain and a thrombospondin type-1 repeat domain. The human recombinant protein was expressed in Drosophila cells and was shown to cleave aggrecan substrate at the aggrecanase cleavage site. The sequences of the three peptides derived from the bovine aggrecanase were encoded within the human cDNA and a cloned portion of bovine cDNA spanning 1579 nucleotides was $94 \%$ homologous to the human cDNA. The enzyme was named aggrecanase- 1 or ADAMTS-4.

\section{Comments}

Loss of cartilage aggrecan has been considered an important event in the progression of arthritis for more than 20 years. The identification and cloning of aggrecanase-1 (and aggrecanase-2) finally provides cartilage biologists with the information they need for much future work to determine the role of these enzymes in normal cartilage turnover and in pathological conditions such as rheumatoid arthritis and osteoarthritis. The availability of recombinant forms of these enzymes is of paramount importance for the identification of specific inhibitors which will be necessary for these studies.

\section{Methods}

Conditioned medium was obtained from IL-1-stimulated bovine nasal cartilage explants over 6 to 18 days of culture. Purification was monitored using purified aggrecan substrate in an in vitro "aggrecanase" assay. Measurement of the aggrecanase-generated neoepitope was by western analysis using monoclonal antibody BC-3. Aggrecanase was purified using a combination of chromatography on Macro $S$ and gelatin agarose followed by affinity purification in two steps using tissue inhibitor of metalloproteinase-1 (TIMP-1) followed by an aggrecanase-inhibitor resin. Purified proteins were subjected to SDS-PAGE and either Coomassie stained or transferred to polyvinylidine difluoride (PVDF) membrane for $\mathrm{N}$-terminal sequencing. Proteins electrophoresed under nonreducing conditions were excised from the gel, dialysed and used in the in vitro "aggrecanase" assay. Using the amino acid sequences determined, a combination of database searching, PCR and rapid amplififcation of cDNA ends (RACE) technology was used to assemble the complete sequence of the human enzyme.

\section{Additional information}

During the course of this work, a second enzyme, aggrecanase-2 or ADAMTS-11, with aggrecanase activity was identified and its gene cloned (Abbaszade I et al, J Biol Chem 1999, 274:23443-23450 [Abstract]). This second enzyme possesses a thrombospondin submotif at its C-terminus which is not present in aggrecanase-1. Excluding the prodomains of aggrecanase-1 and aggrecanase-2, which differ significantly from each other, the remainder of the human protein sequences for these two enzymes are 
$48 \%$ identical. It should be noted that neither of these aggrecanase enzymes are tissue-specific for cartilage.

\section{References}

1. Tortorella MD, Tortorella MD: Purification and cloning of aggrecanase-1: A member of the ADAMTS family of proteins. Science. 1999, 284: 1664-1666.

This PDF file was created after publication. 